Jan Lazar*

Université d'Opole /

Université d'Ostrava
UDK [81'42:355.01(569.1)]:004.738.5

DOI: 10.4312/linguistica.58.1.163-171

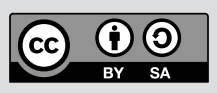

\title{
LA GUERRE EN SYRIE DANS LA PRESSE EN LIGNE : QUELLE EXPRESSIVITÉ DANS LES COMMENTAIRES PUBLICS?
}

\section{INTRODUCTION}

La guerre civile Syrienne, parfois appelée « une révolution syrienne », est un conflit armé toujours en cours qui a éclaté au printemps 2011 en Syrie. Au début, il s'agissait des manifestations pacifiques dont l'objectif était de soutenir la démocratie dans le pays et en même temps de manifester le mécontentement du peuple contre le régime autoritaire du président Bachar el Assad. Néanmoins, les représailles violentes contre les manifestants se sont vite transformées en conflits armés qui ont commencé à ravager tout le pays. Une opposition, formée en exil, s'est donné pour objectif de supprimer le gouvernement de Bachar al Assad et d'installer la démocratie en Syrie. Ses idées ont été soutenues notamment par les pays du Golfe et l'Occident, en particulier par l'Arabie Saoudite, la Turquie, le Qatar et les États-Unis. Le régime autoritaire syrien a été soutenu par l'Iran et la Russie. Il s'ensuit logiquement que le petit conflit national est devenu une guerre internationale qui a commencé à préoccuper plusieurs pays de l'Occident, y compris la France et la République tchèque. D'après les estimations de diverses organisations non-gouvernementales, le conflit a fait autour de 400000 à 500000 morts de mars 2011 à septembre 2016. L'usage des armes chimiques et de nombreux crimes contre l'humanité ont provoqué de nombreuses discussions dans les pays occidentaux qui se sentent, au moins moralement, engagés dans ce conflit. De plus, la guerre en Syrie a fait bouger plusieurs millions de Syriens qui ont été obligés de quitter leurs foyers. En mars 2014, les Nations Unis estimaient que 9 millions des Syriens avaient quitté leurs maisons et plus de 2 millions des gens se sont réfugiés dans les pays voisins. En mai 2014, L'Union européenne a débloqué 50 millions d'euros pour l'aide humanitaire et a essayé de résoudre le problème avec des réfugiés en fixant des quotas pour les pays membres de l'U.E. Ce dernier fait a suscité un grand débat en France ainsi qu'en République tchèque qui devraient accueillir plusieurs milliers de réfugiés. Il nous semble intéressant d'examiner l'expressivité dans les commentaires publics traitant la guerre en Syrie en se demandant si elles contiennent des mots vulgaires ou argotiques. Du fait que nous voulons télécharger notre corpus de commentaires sur Internet, il nous semble important d'ajouter que l'anonymat absolu dans les discussions virtuelles est de plus en plus rare et les internautes sont souvent obligés d'entrer dans le monde virtuel sous pseudonyme. Il convient de préciser que les conditions de création d'un pseudonyme

* jan.lazar@osu.cz 
peuvent sensiblement varier d'un journal en ligne à l'autre, raison pour laquelle certains internautes dévoilent leur identité civile tandis que d'autres la masquent. L'objectif principal de notre recherche est de savoir si la possibilité de commenter anonymement est un facteur important pour la prolifération des mots vulgaires ou argotiques dans les commentaires publics.

\section{IDENTITÉ NUMÉRIQUE DANS L'ESPACE VIRTUEL}

L'une des caractéristiques des environnements numériques est l'anonymat apparent qu'il confère aux locuteurs. On peut parler d'un anonymat visuel car les locuteurs ne se voient pas, mais aussi d'un anonymat discursif, puisque seulement la partie des données numériques est connue aux interlocuteurs. Pourtant, il faut admettre que l'anonymat absolu dans l'espace virtuel reste assez limité et on pourrait plutôt parler du règne du pseudonymat. Paveau (2015) définit le pseudonymat de manière suivante : « Le pseudonymat, pratique aussi ancienne que le port d'un nom, est le choix par un individu d'un autre nom que le sien, quasiment toujours choisi, pour des raisons variées qui ont toutes en commun de masquer son identité officielle. » Il convient de préciser que l'identité numérique qui figure dans notre corpus peut être réparties en 3 catégories principales :

A) Pseudonymes à identité révélée - Ils font référence à l'identité civile des internautes et généralement ils contiennent un anthroponyme complet. Pour illustrer cette catégorie, montrons quelques exemples : Jan Novák, Markéta Spurná, Michel Lardon,

B) Pseudonymes à identité semi-révélée - Ils masquent partiellement l'identité des internautes en relevant seulement quelques indices qui pourraient aider à identifier le locuteur. Ces pseudonymes contiennent habituellement un anthroponyme partiel ou une indice géographique. Voici quelques exemples : HonzikPPP, Janicka, loulou64, mama32

C) Pseudonymes à identité cachée - Il s'agit des pseudonymes qui masquent complètement l'identité de l'internaute. Les pseudonymes peuvent être formés à partir des énoncés variés, jeux de mots, etc. Les exemples suivants illustrent cette catégorie : Jaksemas, tymitaky, commeci,vivelafr

\section{PRÉSENTATION DE NOTRE CORPUS}

Pour constituer notre corpus, nous avons choisi la presse en ligne qui est librement disponible sur Internet et où l'on peut facilement commenter les articles publiés. Au total, nous avons décidé de dépouiller 4 journaux en ligne qui sont bien connus soit en France, soit en République tchèque. Pour obtenir un corpus varié, nous avons sélectionné un journal en ligne plutôt sérieux et un journal en ligne « people». En République tchèque, il s'agit des journaux en ligne Idnes.cz et Blesk.cz. Le premier journal tchèque est destiné au public intellectuel et traite notamment de l'actualité politique dans le pays. Pour pouvoir commenter des articles, les internautes doivent s'enregistrer et dévoiler leur identité en indiquant leur nom et prénom. Les données fournies sont ensuite vérifiées et l'internaute ne peut commencer à commenter les textes qu'après la confirmation finale. 
Le deuxième journal tchèque est plutôt populaire et s'intéresse en premier lieu à la vie privée des stars. Pour pouvoir commenter des articles, les internautes doivent aussi s'enregistrer, mais Blesk est un de rares journaux tchèques qui laisse ses lecteurs choisir leurs pseudonymes et qui n'impose aucune règle pour leur création. Il s'agit d'une stratégie inhabituelle dans le milieu tchèque car la grande majorité des journaux tchèques veut connaitre l'identité de ceux qui commentent leurs textes. En ce qui concerne le corpus français, nous avons choisi pour notre analyse le journal en ligne 20 minutes, qui mélange l'actualité politique avec des informations diverses. Ajoutons qu'il ne force pas ses lecteurs à dévoiler leur identité et ils peuvent aussi choisir librement leur pseudonyme. Le dernier journal de notre corpus, Closer. fr, représente un cas particulier car il autorise ses lecteurs à commenter ses textes dans l'anonymat. Il s'agit d'une stratégie unique dans le milieu francophone car la création d'un pseudonyme est exigée par la majorité écrasante des journaux français. Précisons que les internautes en profitent largement et commentent souvent les textes en anonymat. En ce qui concerne la recherche des articles pour notre corpus, nous nous sommes servi de moteur de recherche où nous avons mis les mots : Válka v Sýrii «La guerre en Syrie ». Nous n'avons sélectionné que des articles qui sont parus comme les derniers publiés avant la date du colloque. La seule exception est le journal Blesk qui sort en premier lieu l'article où il y a une correspondance parfaite avec les mots recherchés.

\section{ANALYSE DE NOTRE CORPUS}

Le premier exemple de notre corpus est représenté par l'article publié le 7 novembre 2016 à 6 : 24 dans le journal Idnes.cz. L'article s'intitule Experti : Až IS padne v Iráku a Sýrii, novou základnu může mít v jižní Asii « Experts : Si EI chute en Iran et en Syrie, il peut créer une nouvelle base au sud de l'Asie». Au total l'article contient 47 commentaires et tous les pseudonymes appartiennent à la première catégorie, puisqu'ils sont composés d'un anthroponyme complet. Il ne s'agit pas d'une décision des internautes, mais c'est une stratégie imposée par le journal Idnes. Son objectif est de dévoiler l'identité de tous les internautes et de bloquer les commentaires vulgaires.

Tableau 1 : Identité numérique sur Idnes.cz

\begin{tabular}{|l|l|l|}
\hline Pseudonymes & Nombre & Expressivité \\
\hline Identité révélée & 47 & 0 \\
\hline Identité semi-révélée & 0 & 0 \\
\hline Identité cachée & 0 & 0 \\
\hline TOTAL & 47 & 0 \\
\hline
\end{tabular}

Comme nous montre l'exemple de notre corpus, cette politique s'avère efficace, puisque les commentaires analysés sont rédigés au registre standard et ne contiennent pas de mots argotiques ou vulgaires. 
Exemple du corpus Idnes.cz :

$\mathrm{Cz}$

Karel Nedopil « Na zapade existuje cela rada oblasti, kde je muslimu uz vetsina. Ted navic podporena cerstvou stotisicovou armadou Merkelovych tricetiletych nezletilych.»

$\mathrm{Fr}$

Karel Nedopil «À l'ouest on retrouve déjà beaucoup de zones où les musulmans sont en majorité. De plus, ils sont soutenus par la jeune armée de Merkel qui contient des centaines de mille de mineurs âgés de 30 ans. »

La deuxième partie de notre corpus tchèque est représentée par l'article Válka $v$ Sýrii už vzala život 7 tisícuim lidi, $40 \%$ z nich jsou ženy a děti!!! « La guerre en Syrie a déjà tué 7 mille personnes, $40 \%$ sont des femmes et des enfants " qui a été publié le 18 août 2016 à $17: 54$ dans le journal Blesk. Comme nous montre le Tableau 2, dès que les internautes tchèques ont la possibilité de masquer leur identité, ils en profitent abondamment. Parmi les 7 pseudonymes analysés, aucun n'appartient à la catégorie de l'indentité révélée.

Tableau 2 : Identité numérique sur Blesk.cz

\begin{tabular}{|l|l|l|}
\hline Pseudonymes & Nombre & Expressivité \\
\hline Identité révélée & 0 & 0 \\
\hline Identité semi-révélée & 1 & 1 \\
\hline Identité cachée & 6 & 4 \\
\hline TOTAL & 7 & 5 \\
\hline
\end{tabular}

L'extrait de notre corpus, nous prouve que la possibilité de masquer l'identité dans les commentaires publics, stimulent les internautes tchèques à l'usage des mots argotiques ou vulgaires. Parmi les 7 commentaires publiés, on retrouve 5 commentaires contenant des mots avec une expressivité forte.

Exemple du corpus Blesk.cz :

$\mathrm{CZ}$

tonny44: Mně to žily netrhá ...Kvưli tomu, že se tam hňupi nedokážou domluvit, by se neměli srráát mezi nás, ale problémy řěsit doma, svviinně ....... . FR

Tonny44: Je m'en fous!.... Comme ces cons ne peuvent pas se mettre d'accord, ils nous emmerdent. Ils devraient résoudre leurs problèmes chez eux! Enfoirés!

En ce qui concerne le corpus français, nous avons analysé au total 44 commentaires. La première partie de notre corpus français contient 31 pseudonymes qui sont apparus 
dans l'article Accusations de crimes de guerre en Syrie: Poutine répond à Hollande (et tacle les Occidentaux) publié le 13 octobre 2016 à $7: 57$ dans le journal 20 minutes. Le Tableau 3 nous montre que les internautes français préfèrent aussi masquer leur identité si le journal en question le permet. La grande majorité des pseudonymes (26) est située dans la dernière catégorie, c'est-à-dire les pseudonymes à identité cachée.

Tableau 3 : Identité numérique sur 20 minutes

\begin{tabular}{|l|l|l|}
\hline Pseudonymes & Nombre & Expressivité \\
\hline Identité révélée & 0 & 0 \\
\hline Identité semi-révélée & 5 & 0 \\
\hline Identité cachée & 26 & 0 \\
\hline TOTAL & 31 & 0 \\
\hline
\end{tabular}

Bien que l'identité numérique sur 20 minutes soit peu contrôlée, on observe que les commentaires sont écrits au registre standard et que les mots argotiques sont absents du corpus.

Exemple du corpus 20minutes:

FR

Serginot : "A chacun «sa» vérité ! Les motivations des uns n'étant pas forcément les motivations des autres. Cependant, une certitude... Ce sont des guerres au détriment des peuples.»

La dernière partie de notre corpus est constituée de 13 commentaires publiés dans le journal Closer. L'article analysé s'intitule «Allah n'a jamais dit de couper des têtes» : Hasna Aït Boulahcen condamnait les agissements de l'Etat islamique » et il était publié le 21 avril 2016 à $16: 46$. Il est à noter que la grande majorité des internautes préfèrent rester anonymes (12) et ne se servent pas de pseudonymes.

Tableau 4 : Identité numérique sur Closer.fr

\begin{tabular}{|l|l|l|}
\hline Pseudonymes & Nombre & Expressivité \\
\hline Identité révélée & 0 & 0 \\
\hline Identité semi-révélée & 0 & 0 \\
\hline Identité cachée & $13(12$ Anonyme $)$ & 0 \\
\hline TOTAL & 13 & 0 \\
\hline
\end{tabular}

Pourtant, il convient de préciser que la possibilité de rester en anonymat absolu n'incite pas les internautes à l'usage des mots vulgaires et argotiques. L'exemple de notre corpus nous montre que les commentaires sont rédigés dans le registre familier, mais ne contiennent pas de mots argotiques. Il est à noter que c'est avant tout le code orthographique qui se voit modifié. Nous observons que la possibilité d'écrire 
anonymement laisse l'expression écrite plus « libre » et les internautes essaient d'approcher le code écrit de l'oral.

Exemple du corpus Closer :

FR

Anonyme : " ben wi, et d' ailleurs c etait une fille bien, tjrs aimable, tjrs bien polie, bien propre... une grande perte »

\section{CONCLUSION}

Notre étude a mis en évidence que les conditions de la création d'un pseudonyme peuvent varier sensiblement d'un pays à l'autre. En République tchèque on observe plutôt la tendance de révéler l'identité des internautes afin de limiter des commentaires comportant des insultes. L'exemple parfait nous fournit le quotidien Idnes.cz qui force ses lecteurs désirant commenter les textes publiés à lui transmettre toutes leurs données personnelles. Chaque internaute doit s'enregistrer et indiquer son nom et prénom pour pouvoir participer aux discussions. D’après nous, le fait de dévoiler l'indetité personnelle est un facteur important qui influence la structure ainsi que le registre des énoncés postés. Notre analyse des commentaires publiés sur Idnes.cz a prouvé que la totalité des énoncés appartenait au registre standard et que l'emploi des mots argotiques était quasiment exclu. Par contre Blesk.cz adopte une stratégie complètement différente et laisse aux internautes libre choix de leurs pseudonymes. Ils en profitent amplement et masquent souvent leur identité sous des pseudonymes variés. On peut constater que la possibilité de masquer l'identité contribue à la prolifération des mots vulgaires et argotiques dans les commentaires publics tchèques. Du fait que la guerre en Syrie suscite beaucoup de polémique, surtout en ce qui concerne les réfugiés, on peut retrouver dans les commentaires une grande expressivité qui se manifeste, entre autres, par l'usage de gros mots tchèques (svině, hňupi). Si on observe le corpus français, on s'aperçoit que les internautes français peuvent choisir librement leur identité numérique. L'analyse de notre corpus montre que la possibilité de cacher leur identité ou de rester anonymes modifie le registre des écrits, mais n'incite pas à l'usage des mots argotiques ou vulgaires. Il pourrait être intéressant de poursuivre cette recherche en analysant d'autres commentaires à propos des sujets polémiques, ce qui nous permettrait de confirmer que l'indentité numérique est décisive pour le choix du registre des énoncés publics.

\section{Bibliographie}

CISLARU, Georgeta (2009) « Le pseudonyme, nom ou discours ? » Les Carnets du Cediscor 11, http://cediscor.revues.org/746

MARTIN, Marcienne (2006) Le pseudonyme sur Internet, une nomination située au carrefour de l'anonymat et de la sphère privée. Paris : Éditions l'Harmattan.

MARTIN, Marcienne (2102) Se nommer pour exister. L'exemple du pseudonyme sur l'internet. Paris : Editions l'Harmattan. 
PAVEAU, Marie-Anne «Les écritures de Protée : identités pseudonymes. » La pensée du discours [carnet de recherche], http://penseedudiscours.hypotheses.org/?p=10057

PAVEAU, Marie-Anne (2015) « Pseudonymat. » Technologies discursives [Carnet de recherche], http://technodiscours.hypotheses.org/?p=245

PEREA, François (2010) «L'identité numérique : de la cité à l'écran. Quelques aspects de la représentation de soi dans l'espace numérique. » Les Enjeux de l'information et de la communication, 144-159.

SCHMITT, Amandine « Avoir un pseudo sur internet sert-il encore à quelque chose ? ", L'Obs [site], http://tempsreel.nouvelobs.com/les-internets/20130726.OBS1203/ avoir-un-pseudo-sur-internet-sert-il-encore-a-quelque-chose.html

THURLOW, Crispin/Laura LENGEL/Alice TOMIC (2004) Computer mediated communication: social interaction and the Internet. Londres : SAGE.

\section{Résumé \\ LA GUERRE EN SYRIE DANS LA PRESSE EN LIGNE : QUELLE EXPRESSI- VITÉ DANS LES COMMENTAIRES PUBLICS?}

La guerre se définit comme une situation conflictuelle entre deux ou plusieurs pays, états, groupes sociaux, individus, avec ou sans lutte armée. Actuellement, la guerre en Syrie préoccupe les médias occidentaux, y compris la presse en ligne. La guerre civile syrienne a éclaté dans le contexte du Printemps arabe par des manifestations majoritairement pacifiques en faveur de la démocratie contre le régime baasiste dirigé par le président Bachar el-Assad. Elle s'est rapidement transformée en rébellion armée qui a exigé l'engagement de certains pays occidentaux, ce qui a provoqué de nombreuses discussions. Dans cette communication nous tentons de comprendre comment le phénomène de la guerre peut intervenir dans les choix de dévoilements identitaires des internautes. Il convient de préciser que le choix du pseudonyme est l'un des premiers actes de création individuelle de l'internaute : avant de s'exprimer par la langue, il va devoir se nommer. L'objectif de notre article est d'analyser plus en détail quel est le lien entre l'expressivité des commentaires et l'identité numérique. Nous voulons aussi examiner si la possibilité de masquer l'identité est un facteur déclencheur de l'usage des mots argotiques. Pour répondre à ces questions nous proposons une étude comparative de deux corpus de commentaires publics apparaissant sur des sites de presse en ligne qui décrivent la guerre en Syrie. Le premier corpus sera constitué sur la base de sites tchèques (Idnes.cz, Blesk.cz), le second sur la base de sites français (20.minutes.fr, Closer.fr), selon un protocole identique.

Mots-clés : guerre, Syrie, expressivité, commentaire public 


\section{Abstract \\ THE WAR IN SYRIA IN ONLINE PRESS: WHAT EXPRESSIVENESS IS THERE IN PUBLIC COMMENTS?}

A war is defined as a conflict between two or more countries, states, social groups, individuals, with or without an armed struggle. Currently the Western media, including online press, are very interested in the Syrian war. The Syrian civil war broke out in the context of the Arab Spring, starting with mostly peaceful demonstrations in favour of democracy and against the Baathist regime led by president Bashar al-Assad. It quickly turned into an armed rebellion that demanded the intervention of some Western countries, thus provoking much discussion. In this paper we try to understand how the phenomenon of war can intervene in the choice of identity disclosure of internet users. It should be pointed out that the choice of a username is one of the first acts undertaken by an internet user, and that before expressing oneself one has to identify oneself. The aim of this article is to analyse the relationship between the language use in comments and the digital identity of their producers. We also examine whether the possibility of hiding one's true identity is a trigger factor for the use of slang words. To answer these questions we propose a comparative study of two corpora of public commentaries appearing in online press sites that describe the war in Syria. The first corpus consists of Czech sites (Idnes. cz, Blesk.cz), whereas the second one is made up of French sites (20.minutes.fr, Closer.fr), according to an identical protocol.

Keywords : war, Syria, expressivity, public comments

\section{Povzetek}

\section{VOJNA V SIRIJI V SPLETNIH MEDIJIH: KAKŠEN JE ODZIV JAVNOSTI V SPLETNIH KOMENTARJIH?}

Vojno definiramo kot konfliktno stanje med dvema ali več državami, skupnostmi, socialnimi skupinami, posamezniki, pri čemer ni nujno, da pride do oboroženih spopadov. Vojna v Siriji je v ospredju pozornosti zahodnih medijev, med katere spadajo tudi spletni mediji. Državljanska vojna v Siriji se je začela med arabsko pomladjo z demonstracijami, v večini miroljubnimi, ki so se prizadevale za vzpostavitev demokratičnega sistema in bile usmerjene proti vladajočemu režimu predsednika Bašarja el Asada. Vendar se je hitro spremenila v oborožen upor, ki je terjal posredovanje zahodnih sil, kar je vodilo $\mathrm{v}$ številne razprave. $\mathrm{V}$ pričujoči študiji raziskujemo, kako vojna vpliva na razkrivanje idenitite internetnih uporabnikov. Izbira psevdonima je prvo kreativno dejanje uporabnika, saj se mora, preden se začne izražati, poimenovati. Cilj naše raziskave je bolj poglobljena analiza povezave med ekspresivnostjo komentarjev in digitalno identiteto. Prav tako poskušamo analizirati, ali je možnost skrivanja identitete dejavnik, ki spodbuja k uporabi argojevskih 
besed. Da bi odgovorili na zastavljeni vprašanji, predstavljamo primerjalno analizo dveh korpusov komentarjev uporabnikov spletnih časopisnih strani, ki se dotikajo vojne v Siriji. Prvi korpus je sestavljen iz čeških spletnih virov (Idnes.cz, Blesk. cz), drugi pa iz francoskih virov sorodnega tipa (20.minutes.fr, Closer.fr).

Ključne besede: vojna, Sirija, ekspresivnost, javno izražanje komentarjev 